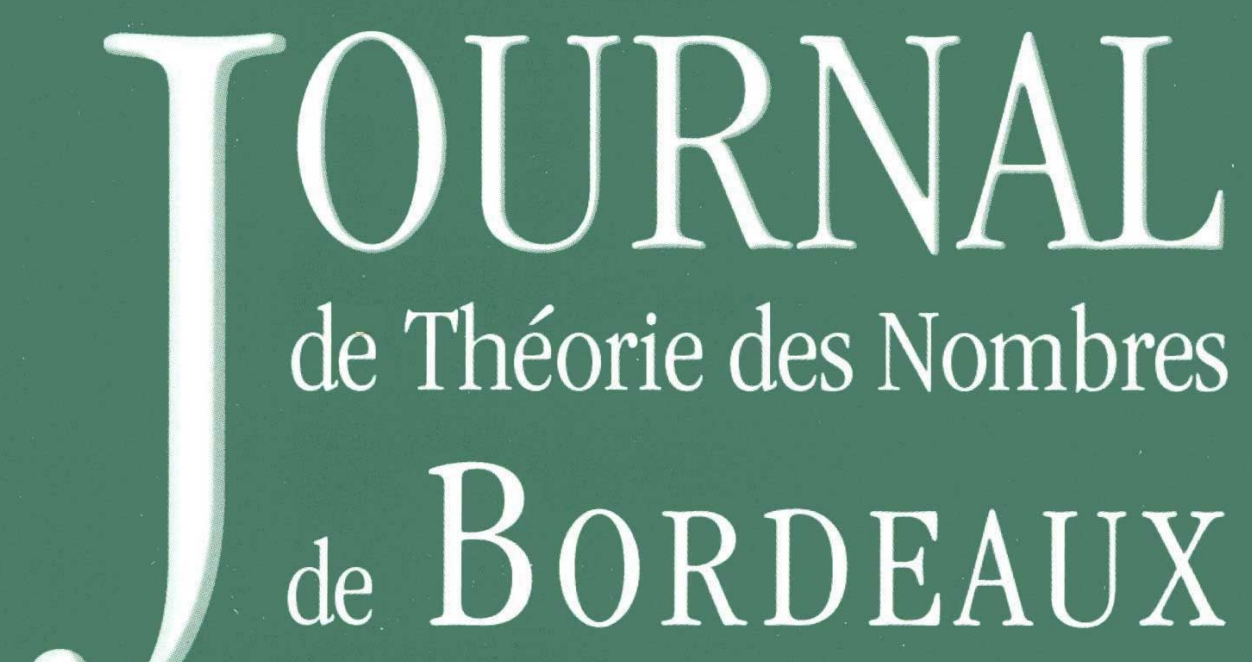

anciennement Séminaire de Théorie des Nombres de Bordeaux

\title{
Romain VALIDIRE
}

Capitulation for even $\boldsymbol{K}$-groups in the cyclotomic $\mathbb{Z}_{\boldsymbol{p}}$-extension.

Tome 21, no 2 (2009), p. 439-454.

<http://jtnb.cedram.org/item?id=JTNB_2009__21_2_439_0>

(C) Université Bordeaux 1, 2009, tous droits réservés.

L'accès aux articles de la revue «Journal de Théorie des Nombres de Bordeaux » (http://jtnb.cedram.org/), implique l'accord avec les conditions générales d'utilisation (http://jtnb.cedram. org/legal/). Toute reproduction en tout ou partie cet article sous quelque forme que ce soit pour tout usage autre que l'utilisation à fin strictement personnelle du copiste est constitutive d'une infraction pénale. Toute copie ou impression de ce fichier doit contenir la présente mention de copyright.

\section{cedram}




\title{
Capitulation for even $K$-groups in the cyclotomic $\mathbb{Z}_{p^{-} \text {-extension. }}$
}

\author{
par ROMAIN VALIDIRE
}

RÉSUMÉ. Soit $p$ un nombre premier et $F$ un corps de nombres. Depuis les travaux d'Iwasawa, le comportement de la $p$-partie du groupe des classes d'idéaux dans une $\mathbb{Z}_{p}$-extension de $F$ est assez bien compris. M. Grandet et J.-F. Jaulent ont en outre donné un résultat précis concernant sa structure de groupe abélien.

Par ailleurs, le groupe des classes d'idéaux s'interprête comme la partie de torsion du $K_{0}$ de l'anneau des entiers de $F$. Les $K$ groupes pairs de l'anneau des entiers peuvent être vus comme des versions supérieures du groupe des classes et le comportement de ces $K$-groupes dans les $\mathbb{Z}_{p}$-extensions a déjà été étudié par de nombreux auteurs. Dans cet article, nous montrons que le résultat de Grandet et Jaulent sur les groupes de classes est encore vrai pour les $K$-groupes pairs dans la $\mathbb{Z}_{p}$-extension cyclotomique.

Abstract. Let $p$ be a prime number and $F$ be a number field. Since Iwasawa's works, the behaviour of the $p$-part of the ideal class group in the $\mathbb{Z}_{p}$-extensions of $F$ has been well understood. Moreover, M. Grandet and J.-F. Jaulent gave a precise result about its abelian $p$-group structure.

On the other hand, the ideal class group of a number field may be identified with the torsion part of the $K_{0}$ of its ring of integers. The even $K$-groups of rings of integers appear as higher versions of the class group. Many authors have already studied the behaviour of the higher even $K$-groups in a $\mathbb{Z}_{p}$-extension. Here, we prove that Grandet and Jaulent's result on class group still holds for higher even $K$-groups in the cyclotomic $\mathbb{Z}_{p}$-extension.

\section{Introduction}

Let $p$ be a prime number and $F$ be a number field. We denote by $\mathcal{O}_{F}$ the ring of integers of $F$ and by $C l\left(\mathcal{O}_{F}\right)$ the ideal class group of $\mathcal{O}_{F}$.

Let $F_{\infty} / F$ be a $\mathbb{Z}_{p}$-extension, with finite layers $F_{n}$ for all integers $n$ and with the usual notations for the Galois groups $\Gamma:=\operatorname{Gal}\left(F_{\infty} / F\right)$ and $\Gamma_{n}:=$ $\operatorname{Gal}\left(F_{\infty} / F_{n}\right)$. Iwasawa's theory of $\mathbb{Z}_{p}$-extensions is a way to investigate the behaviour of the $p$-primary part of the class groups $C l\left(\mathcal{O}_{F_{n}}\right)$. A well-known result is the famous Iwasawa's formula giving the order of the $p$-primary part of $C l\left(\mathcal{O}_{F_{n}}\right)$ for all $n$ large enough. It is possible to obtain more precise 
results about this group by studying the vanishing of ideal classes in the $\mathbb{Z}_{p}$-extension. Let us consider the natural map :

$$
C l\left(\mathcal{O}_{F_{n}}\right)\{p\} \rightarrow\left(C l\left(\mathcal{O}_{F_{\infty}}\right)\{p\}\right)^{\Gamma_{n}}
$$

between the $p$-primary part of $C l\left(\mathcal{O}_{F_{n}}\right)$ and the $\Gamma_{n}$-fixed points of the inductive limit:

$$
C l\left(\mathcal{O}_{F_{\infty}}\right)\{p\}:=\lim _{\rightarrow} C l\left(\mathcal{O}_{F_{n}}\right)\{p\} .
$$

We denote by $\operatorname{Cap}\left(F_{n}\right)$ the kernel of this map. These capitulation kernels have been intensively studied (cp. $[\mathrm{Iw}],[\mathrm{Ku}], \ldots)$ and their asymptotical behaviour is well-known: for $m \geq n \gg 0$ the norm map between the class groups induces an isomorphism from $\operatorname{Cap}\left(F_{m}\right)$ to $\operatorname{Cap}\left(F_{n}\right)(\mathrm{cp} .[\mathrm{Ku}])$; we say that the capitulation kernels stabilize for the norm map.

Consider the Iwasawa module $X_{F_{\infty}}:=\lim C l\left(\mathcal{O}_{F_{m}}\right)\{p\}$, where the limit is taken with respect to the norm maps. This group is a module over the complete group ring $\mathbb{Z}_{p}[[\Gamma]]$. Let $\mu$ and $\lambda$ be respectively the $p$-valuation and the degree of the characteristic polynomial of $X_{F_{\infty}}$. For $n$ sufficiently large, the natural map from $X_{F_{\infty}}$ to $C l\left(\mathcal{O}_{F_{n}}\right)\{p\}$ induces an isomorphism from $\left(X_{F_{\infty}}\right)^{0}$ to $\operatorname{Cap}\left(F_{n}\right)$, where $\left(X_{F_{\infty}}\right)^{0}$ is the maximal finite submodule of $X_{F_{\infty}}$.

However, we have a more precise result; M. Grandet and J.-F. Jaulent prove in [GJ] that the capitulation kernel becomes a direct summand of the class group:

Theorem 0.1. Assume that the invariant $\mu$ of $X_{\infty}$ is trivial. Then there exists $\left(\alpha_{1}, \ldots, \alpha_{\lambda}\right) \in \mathbb{Z}^{\lambda}$ such that for all $n$ large enough:

$$
C l\left(\mathcal{O}_{F_{n}}\right)\{p\} \simeq \operatorname{Cap}\left(F_{n}\right) \oplus\left(\bigoplus_{i=1}^{\lambda} \mathbb{Z} / p^{\alpha_{i}+n}\right) \text {, as abelian groups. }
$$

On the other hand it is well-known that $C l\left(\mathcal{O}_{F}\right)$ may be identified with the torsion part of $K_{0}\left(\mathcal{O}_{F}\right)$. As for class group we can consider the following higher capitulation kernels for all integers $i \geq 1$ and $n \geq 1$ :

$$
\operatorname{Cap}_{i}\left(F_{n}\right):=\operatorname{ker}\left(K_{2 i}\left(\mathcal{O}_{F_{n}}\right) \otimes \mathbb{Z}_{p} \rightarrow\left(K_{2 i}\left(\mathcal{O}_{F_{\infty}}\right) \otimes \mathbb{Z}_{p}\right)^{\Gamma_{n}}\right),
$$

where $K_{2 i}\left(\mathcal{O}_{F_{n}}\right)$ denotes the Quillen $K$-groups associated with the ring $\mathcal{O}_{F_{n}}$ and

$$
K_{2 i}\left(\mathcal{O}_{F_{\infty}}\right):=\lim _{\rightarrow} K_{2 i}\left(\mathcal{O}_{F_{m}}\right) .
$$

Using a general result due to T. Nguyen Quang Do, B. Kahn proved (cp. $[\mathrm{Ka}])$ that the groups $\operatorname{Cap}_{1}\left(F_{n}\right)$ also stabilize for the norm map.

Now we assume that $p$ is odd or $p=2$ and $F$ contains $\sqrt{-1}$. For a finite set $S$ of primes containing the set of primes above $p$ and the infinite primes of $F$, let $\mathcal{O}_{F}^{S}$ denote the ring of $S$-integers of $F$. Generalizing the result of $\mathrm{T}$. 
Nguyen Quang do and B. Kahn, M. Kolster and A. Movahhedi introduced (cp. $[\mathrm{KM}]$ ) similar capitulation kernels $\operatorname{Cap}_{i}^{e ́ t}\left(F_{n}\right)$ for all $i \geq 1$ using étale $K$-groups $K_{2 i}^{e ́ t}\left(\mathcal{O}_{F_{n}}^{S}\right)$ and proved for these groups the same stabilization property.

Our purpose in the present article is to prove that the theorem $(0.1)$ also holds for higher étale capitulation kernels when $F_{\infty}$ is the cyclotomic $\mathbb{Z}_{p}$-extension of $F$.

To prove the result, we first consider a particular subgroup of $K_{2 i}^{e ́ t}\left(\mathcal{O}_{F}^{S}\right)$ : the étale wild kernel denoted by $W K_{2 i}^{e ́ t}(F)$. The definition of the wild kernels is given in section 2 .

In section 3, we use a description of $W K_{2 i}^{e ́ t}(F)$ due to Schneider to prove that the capitulation kernels for the wild kernels also become direct summands.

In section 4 , we prove that the $p$-quotients of the wild kernels and of the $p$-class group are asymptotically isomorphic (Proposition 4.1). Then we use this result to show that, when $\mu=0$, the group $\operatorname{Cap}_{i}^{e t}\left(F_{n}\right)$ becomes a direct summand of the abelian $p$-group $K_{2 i}^{e t}\left(\mathcal{O}_{F_{n}}^{S}\right)$. Finally, we show that we have a non canonical Galois descent for the even $K$-groups in the cyclotomic $\mathbb{Z}_{p}$-extension (Corollary 4.2).

Acknowledgements. The author would like to thank J.-F. Jaulent and A. Movahhedi for many helpful comments. The author also expresses his acknowledgements to M. Kolster and to T. Nguyen Quang Do whose remarks improved an earlier version of the paper.

\section{Preliminaries}

In this section we introduce the objects studied in the rest of the paper. First, we fix some notations.

Let $p$ be a fixed prime number and $F$ be an algebraic number field. If $p=2$ we also assume that $\sqrt{-1} \in F$. Let $S$ be a finite set of primes in $F$, containing the set $S_{p}$ of primes above $p$ and the set $S_{\infty}$ of infinite primes; let $\mathcal{O}_{F}^{S}$ denote the ring of $S$-integers of $F$ and $G_{F}^{S}$ denote the Galois group over $F$ of the maximal algebraic extension of $F$ which is unramified outside $S$. For any $\mathbb{Z}_{p}$-module $M$, we put $M^{*}=\operatorname{Hom}\left(M, \mathbb{Q}_{p} / \mathbb{Z}_{p}\right)$, the Pontrjagin dual of $M$.

For integers $n \geq 0$ and $i \geq 1$ we denote by $\mu_{n}^{\otimes i}$ the $i$ th twist of the group $\mu_{n}$ of roots of unity of order $n$ and $\mathbb{Z}_{p}(i):=\varliminf_{\varlimsup}\left(\mu_{p^{n}}^{\otimes i}\right)$, the $i$ th twist of $\mathbb{Z}_{p}$. For any arbitrary $\mathbb{Z}_{p}\left[G_{F}^{S}\right]$-module $M$, we define the $i$-fold Tate twist of $M$ by:

$$
M(i):=M \otimes_{\mathbb{Z}_{p}} \mathbb{Z}_{p}(i) .
$$

For an abelian group $A$ and a positive integer $n$ we denote by:

- $A / n$, the quotient of $A$ by the subgroup $n A$. 
- ${ }_{n} A$, the group of elements $a \in A$ such that $n a=0$.

- $A\{p\}=\bigcup_{k \geq 1}\left(p^{k} A\right)$, the $p$-primary part of $A$.

For $i \geq 1$ and $k=1$ or 2 , the higher étale $K$-theory groups $K_{2 i+2-k}^{e ́}\left(\mathcal{O}_{F}^{S}\right)$, introduced by Dwyer and Friedlander ([DF]), coincide with the (continuous) Galois cohomology groups:

$$
\begin{aligned}
K_{2 i+2-k}^{e ́ t}\left(\mathcal{O}_{F}^{S}\right) & \simeq H^{k}\left(G_{F}^{S}, \mathbb{Z}_{p}(i+1)\right) \\
& \simeq \varliminf_{\longleftarrow} H^{k}\left(G_{F}^{S}, \mathbb{Z} / p^{n}(i+1)\right) .
\end{aligned}
$$

Using Borel's results on algebraic $K$-groups, it can be shown that the even $K$-groups $K_{2 i}^{e t}\left(\mathcal{O}_{F}^{S}\right)$ are finite and that the odd $K$-groups $K_{2 i+1}^{e ́ t}\left(\mathcal{O}_{F}^{S}\right)$ are finitely generated over $\mathbb{Z}_{p}$; their $\mathbb{Z}_{p}$-rank is $r_{1}+r_{2}$ if $i$ is even and $r_{2}$ if $i$ is odd. As usual $r_{1}$ (resp. $r_{2}$ ) denotes the number of real (resp. pairs of conjugate complex) embeddings of $F$. Furthermore the odd étale $K$ groups do not depend on the choice of the set $S$. We have $K_{2 i+1}^{e t}\left(\mathcal{O}_{F}^{S}\right) \simeq$ $H^{1}\left(F, \mathbb{Z}_{p}(i+1)\right)$, and we denote these groups by $K_{2 i+1}^{e t}(F)$.

For any group $G$ and any $G$-module $M$, we denote as usual by $M^{G}$ the fixed points of $M$ under the action of $G$ and by $M_{G}$ the quotient of $M / I_{G} M$, where $I_{G}$ is the augmentation-ideal of $\mathbb{Z}[G]$.

Let $L$ be a finite Galois extension of $F$ with Galois group $G$, which is unramified outside $S$. We are interested in Galois descent and co-descent for étale odd $K$-groups in the extension $L / F$; we have two canonical morphisms between $K_{2 i}^{e ́ t}\left(\mathcal{O}_{F}^{S}\right)$ and $K_{2 i}^{e ́ t}\left(\mathcal{O}_{L}^{S}\right)$ :

- the extension map $K_{2 i}^{e t}\left(\mathcal{O}_{F}^{S}\right) \rightarrow K_{2 i}^{e ́ t}\left(\mathcal{O}_{L}^{S}\right)^{G}$, which may be identified with the restriction map in Galois cohomology.

- the norm map $K_{2 i}^{e ́ t}\left(\mathcal{O}_{L}^{S}\right)_{G} \rightarrow K_{2 i}^{e ́ t}\left(\mathcal{O}_{F}^{S}\right)$, which may be identified with the co-restriction map in Galois cohomology.

We have the following (see $[\mathrm{Ka}]$ and $[\mathrm{KM}]$ ):

Theorem 1.1. Let $L / F$ be a Galois p-extension with Galois group G. Let $S$ be a finite set of primes, containing the primes above $p$ and the primes which ramify in $L$. Then for $i \geq 1$ there is an exact sequence induced by the extension map:

$$
\begin{aligned}
0 \rightarrow H^{1}\left(G, K_{2 i+1}^{e ́ t}(L)\right) & \rightarrow K_{2 i}^{e ́ t}\left(\mathcal{O}_{F}^{S}\right) \\
& \rightarrow K_{2 i}^{e ́ t}\left(\mathcal{O}_{L}^{S}\right)^{G} \rightarrow H^{2}\left(G, K_{2 i+1}^{e t}(L)\right) \rightarrow 0
\end{aligned}
$$

and an isomorphism induced by the norm map:

$$
K_{2 i}^{e ́ t}\left(\mathcal{O}_{L}^{S}\right)_{G} \simeq K_{2 i}^{e ́ t}\left(\mathcal{O}_{F}^{S}\right) .
$$

We deduce the following corollary: 
Corollary 1.1. The kernel of the extension map

$$
\operatorname{Cap}_{i}(L / F):=\operatorname{ker}\left(K_{2 i}^{e ́ t}\left(\mathcal{O}_{F}^{S}\right) \rightarrow K_{2 i}^{e ́ t}\left(\mathcal{O}_{L}^{S}\right)\right)
$$

does not depend on the set $S$ containing $S_{p} \cup S_{\infty}$ and the ramified primes in $L / F$.

Let $F_{\infty} / F$ be an arbitrary $\mathbb{Z}_{p}$-extension with finite layers $F_{n}$. For integers $m \geq n \geq 0$, we put $G_{m, n}=\operatorname{Gal}\left(F_{m} / F_{n}\right)$ and $\Gamma_{n}=\operatorname{Gal}\left(F_{\infty} / F_{n}\right)$. The main objects studied here are the kernels of the extension maps:

$$
\operatorname{Cap}_{i}\left(F_{m} / F_{n}\right):=\operatorname{ker}\left(K_{2 i}^{e ́ t}\left(\mathcal{O}_{F_{n}}^{S}\right) \rightarrow K_{2 i}^{e ́ t}\left(\mathcal{O}_{F_{m}}^{S}\right)^{G_{m, n}}\right) \text {. }
$$

Remark. Since a $\mathbb{Z}_{p}$-extension of number field is $p$-ramified, it is enough to consider the case $S=S_{p} \cup S_{\infty}$; we put $\mathcal{O}_{F}^{\prime}:=\mathcal{O}_{F}^{S_{p} \cup S_{\infty}}$.

We consider the kernel: $\operatorname{Cap}_{i}\left(F_{n}\right):=\operatorname{ker}\left(K_{2 i}^{e ́ t}\left(\mathcal{O}_{F_{n}}^{S}\right) \rightarrow K_{2 i}^{e ́ t}\left(\mathcal{O}_{F_{\infty}}^{S}\right)^{\Gamma_{n}}\right)$, with :

$$
K_{2 i}^{e ́ t}\left(\mathcal{O}_{F_{\infty}}^{S}\right):=\lim _{\rightarrow} K_{2 i}^{e ́ t}\left(\mathcal{O}_{F_{m}}^{S}\right) .
$$

We may deduce from theorem 1.1 the short exact sequence (see [Ka] and $[\mathrm{KM}])$ :

$$
0 \rightarrow \operatorname{Cap}_{i}\left(F_{n}\right) \rightarrow K_{2 i}^{e ́ t}\left(\mathcal{O}_{F_{n}}^{S}\right) \rightarrow K_{2 i}^{e ́ t}\left(\mathcal{O}_{F_{\infty}}^{S}\right)^{\Gamma_{n}} \rightarrow 0 .
$$

Now, we focus on the asymptotical behaviour of the higher capitulation kernels. We have the following proposition:

Proposition 1.1. For all $m \geq n \gg 0$, the Galois group $G_{m, n}$ acts trivially on $\operatorname{Cap}_{i}\left(F_{m}\right)$ and the norm map induces an isomorphism:

$$
\operatorname{Cap}_{i}\left(F_{m}\right)=\operatorname{Cap}_{i}\left(F_{m}\right)_{G_{m, n}} \simeq \operatorname{Cap}_{i}\left(F_{n}\right) \text {. }
$$

Our purpose is to prove that the exact sequence (1.1) is a split exact sequence of abelian groups when $F_{\infty}$ is the cyclotomic $\mathbb{Z}_{p}$-extension.

We need the following lemma on abelian groups:

Lemma 1.1. Let $M$ be a finite abelian p-group and $N$ be a subgroup. Let $e$ be an integer such that $p^{e}$ annihilates $N$. If for all integers $n, 0 \leq n \leq e$, the inclusion map from $N$ to $M$ induces an injection $N / p^{n} \hookrightarrow M / p^{n}$ then $N$ is a direct summand in $M$.

\section{Localisation kernels and étale wild kernels}

In the following, we will consider the localisation kernels for $i \in \mathbb{Z}$ and $n \geq 1$ :

$$
\begin{aligned}
\amalg_{S}^{2}\left(F, \mathbb{Z} / p^{n}(i)\right) & :=\operatorname{ker}\left(H^{2}\left(G_{F}^{S}, \mathbb{Z} / p^{n}(i)\right) \stackrel{\text { loc. }}{\rightarrow} \oplus_{v \in S} H^{2}\left(F_{v}, \mathbb{Z} / p^{n}(i)\right)\right), \\
\amalg_{S}^{2}\left(F, \mathbb{Z}_{p}(i)\right): & =\operatorname{ker}\left(H^{2}\left(G_{F}^{S}, \mathbb{Z}_{p}(i)\right) \stackrel{\text { loc. }}{\rightarrow} \oplus_{v \in S} H^{2}\left(F_{v}, \mathbb{Z}_{p}(i)\right)\right) .
\end{aligned}
$$


P. Schneider studied these localisation kernels in $[\mathrm{S}]$. He conjectured that for all $i \in \mathbb{Z}$, the groups $\amalg_{S}^{2}\left(F, \mathbb{Z}_{p}(i)\right)$ are finite (indeed, it is true for $i \geq 1$ ). For $i=0$, the finiteness of $\amalg_{S}^{2}\left(F, \mathbb{Z}_{p}\right)$ is equivalent to the famous Leopoldt Conjecture.

Let us give some interpretations for these kernels.

Proposition 2.1. (see [NSW, Lemma (8.6.3)]) The groups $\amalg_{S}^{2}\left(F, \mathbb{Z} / p^{n}(i)\right)$ are finite and for $i=1$ we have the isomorphism:

$$
C l\left(\mathcal{O}_{F}^{S}\right) / p^{n} \simeq \amalg_{S}^{2}\left(F, \mu_{p^{n}}\right) .
$$

Remark. By finiteness of class group $\amalg_{S}^{2}\left(F, \mathbb{Z}_{p}(1)\right) \simeq C l\left(\mathcal{O}_{F}^{S}\right)\{p\}$.

Let $n$ be a positive integer. Since $p$ is an odd prime (or $\sqrt{-1} \subset F$ if $p=2$ ) we have $c d_{p}\left(G_{F}^{S}\right) \leq 2$ (cohomological $p$-dimension); the exact cohomology sequence of the short exact sequence

$$
0 \rightarrow \mathbb{Z}_{p}(i+1) \rightarrow \mathbb{Z}_{p}(i+1) \rightarrow \mathbb{Z} / p^{n}(i+1) \rightarrow 0
$$

yields the isomorphism:

$$
H^{2}\left(G_{F}^{S}, \mathbb{Z}_{p}(i+1)\right) / p^{n} \simeq H^{2}\left(G_{F}^{S}, \mathbb{Z} / p^{n}(i+1)\right),
$$

whence follows (see [Ta, Theorem (6.2)]):

Proposition 2.2. Assume that $F$ contains $\mu_{p^{n}}$. Then for $i \geq 1$, we have a canonical isomorphism:

$$
K_{2 i}^{e ́ t}\left(\mathcal{O}_{F}^{S}\right) / p^{n} \simeq H^{2}\left(G_{F}^{S}, \mu_{p^{n}}\right)(i),
$$

and an exact sequence:

$$
0 \rightarrow C l\left(\mathcal{O}_{F}^{S}\right) / p^{n}(i) \rightarrow K_{2 i}^{e t}\left(\mathcal{O}_{F}^{S}\right) / p^{n} \stackrel{\left.\oplus l_{v}\right)}{\longrightarrow} \bigoplus_{v \in S} \mu_{p^{n}}(i-1) \stackrel{\Sigma}{\rightarrow} \mu_{p^{n}}(i-1) \rightarrow 0,
$$

where $l_{v}$ comes from the localisation map at the prime $v \in S$ and $\Sigma$ is the product map.

Tate's results ([Ta]) on $K_{2}$ and Galois cohomology give a canonical isomorphism:

$$
W K_{2}(F)\{p\} \simeq \amalg_{S}^{2}\left(F, \mathbb{Z}_{p}(2)\right),
$$

where $W K_{2}(F)$ is the classical wild kernel (i.e. the kernel of all Hilbert symbols on $\left.K_{2}(F)\right)$ which appears in Moore's exact sequence:

$$
0 \rightarrow W K_{2}(F) \rightarrow K_{2}(F) \rightarrow \oplus_{v} \mu\left(F_{v}\right) \rightarrow \mu(F) \rightarrow 0,
$$

where $v$ runs through all finite and real infinite primes of $F$, and $\mu(F)$ (resp. $\mu\left(F_{v}\right)$ ) denotes the group of roots of unity of the number field $F$ (resp. the local field $F_{v}$ ).

The groups $\amalg_{S}^{2}\left(F, \mathbb{Z}_{p}(i+1)\right)$ do not depend on the choice of the set $S$ containing $S_{p} \cup S_{\infty}$. For all $i \geq 1$, they can be identified with subgroups 
of $K_{2 i}^{e t}\left(\mathcal{O}_{F}^{S}\right)$. Thus, these remarks and the description of the classical wild kernel leads to the definition of the higher étale wild kernels (cp. [Ba], [N2]).

Definition. Let $p$ be a prime number. For a number field $F$ and $i \geq 1$, we define the $2 i$ th étale wild kernel:

$$
W K_{2 i}^{e ́ t}(F):=\amalg_{S}^{2}\left(F, \mathbb{Z}_{p}(i+1)\right) .
$$

The Poitou-Tate duality sequence yields the short exact sequence:

$$
\begin{aligned}
0 \rightarrow W K_{2 i}^{e t}(F) \rightarrow K_{2 i}^{e ́ t}\left(\mathcal{O}_{F}^{S}\right) \rightarrow \oplus_{v \in S} H^{2}\left(F_{v},\right. & \left.\mathbb{Z}_{p}(i+1)\right) \\
& \rightarrow H^{0}\left(F, \mathbb{Q}_{p} / \mathbb{Z}_{p}(-i)\right)^{*} \rightarrow 0 .
\end{aligned}
$$

There exist some relations between the étale wild kernels when $i$ varies over the positive integers. For example we have (cp. [N3])

Proposition 2.3. Assume that $F$ contains $\mu_{p^{n}}$. For all positive integers $i$ and $j$ there is a canonical isomorphism:

$$
W K_{2 i}^{e ́ t}(F) / p^{n} \simeq W K_{2 j}^{e ́ t}(F) / p^{n}(i-j) .
$$

Let us point out another map which will be useful in the following. For a number field $F$ containing $\mu_{p^{n}}$ and for $i \geq 1$, the isomorphism (2.1) yields the commutative diagram:

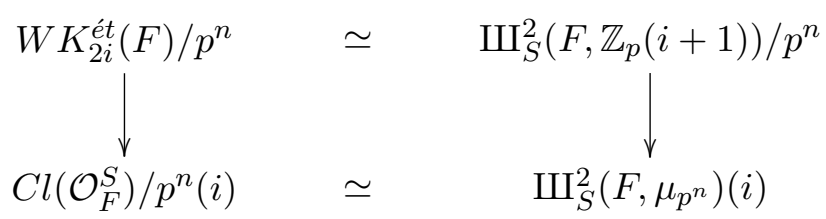

The vertical maps are in general not bijective; in the last section we will give conditions for bijectivity. For the moment, let us give a condition for surjectivity (see [KM, Lemma 2.8] and [V, Proposition 1.3.8]).

Proposition 2.4. Assume that $F$ contains $\mu_{p^{n}}$ and that at least one $p$-adic prime in $F$ totally ramifies in $F_{\infty} / F$. For all $i \geq 1$, the maps

$$
W K_{2 i}^{e ́ t}(F) / p^{n} \rightarrow C l\left(\mathcal{O}_{F}^{S}\right) / p^{n}(i)
$$

are onto.

Remark. It is also possible to construct these maps passing through the logarithmic valuations and the logarithmic class group $\widetilde{\mathcal{C}} \ell(F)$ introduced by Jaulent (cp. [J1] and [J2]). 


\section{Iwasawa theory for wild kernels and capitulation}

In this section we study the capitulation kernel for étale wild kernels when $F_{\infty} / F$ is the cyclotomic $\mathbb{Z}_{p}$-extension of $F$. As usual we put:

$$
W K_{2 i}^{e ́ t}\left(F_{\infty}\right):=\lim _{\rightarrow} W K_{2 i}^{e ́ t}\left(F_{n}\right) .
$$

For all $m \geq n \geq 0$ we have the following equalities:

$$
\begin{aligned}
\operatorname{Cap}_{i}\left(F_{m} / F_{n}\right) & =\operatorname{ker}\left(W K_{2 i}^{e ́ t}\left(F_{n}\right) \rightarrow W K_{2 i}^{e ́ t}\left(F_{m}\right)\right), \\
\operatorname{Cap}_{i}\left(F_{n}\right) & =\operatorname{ker}\left(W K_{2 i}^{e ́ t}\left(F_{n}\right) \rightarrow W K_{2 i}^{e ́ t}\left(F_{\infty}\right)\right)
\end{aligned}
$$

Then the capitulation kernels for wild kernels stabilize for the norm map in the same manner as for odd $K$-groups.

We may deduce from proposition(1.1) (see also [LMN, Lemma 1.1]) that for $i \geq 1$ there is a short exact sequence:

$$
0 \rightarrow \operatorname{Cap}_{i}\left(F_{n}\right) \rightarrow W K_{2 i}^{e ́ t}\left(F_{n}\right) \rightarrow W K_{2 i}^{e ́ t}\left(F_{\infty}\right)^{\Gamma_{n}} \rightarrow 0 .
$$

Following the ideas of Grandet and Jaulent we prove that, under certain assumptions, (3.1) is a split exact sequence of abelian groups.

We still assume that $p$ is odd and $\sqrt{-1} \in F$, if $p=2$. Let $E=F\left(\mu_{2 p}\right)$ and $E_{\infty}$ be the cyclotomic $\mathbb{Z}_{p}$-extension of $E$. We still denote by $\Gamma$ (resp. $\left.\Gamma_{n}\right)$ the Galois group of $E_{\infty} / E\left(\operatorname{resp} . E_{n} / E\right)$. Let $\Delta=\operatorname{Gal}(E / F)$ and let $d$ be the order of $\Delta$.

Now let us give the description of étale wild kernels using Iwasawa theory (cp.[N3]).

We put $X_{\infty}^{\prime}:=\lim C l\left(\mathcal{O}_{E_{n}}^{\prime}\right)\{p\}$, where the limit is taken for the norm map. The $\mathbb{Z}_{p}$-module $X_{\infty}^{\prime}$ is naturally a module over the complete group $\operatorname{ring} \Lambda:=\mathbb{Z}_{p}[[\Gamma]] \simeq \mathbb{Z}_{p}[[1-\gamma]]$, for any chosen topological generator $\gamma$ of $\Gamma$.

Let $L_{\infty}^{\prime}$ be the maximal abelian unramified pro-p-extension of $E_{\infty}$, in which all primes above $p$ are completely decomposed. By class field theory $X_{\infty}^{\prime}$ is isomorphic to the Galois group $\operatorname{Gal}\left(L_{\infty}^{\prime} / E_{\infty}\right)$.

One shows that $X_{\infty}^{\prime}$ is a finitely generated $\Lambda$-torsion module. Let $f(1-$ $\gamma$ ) be its characteristic polynomial. We denote by $\mu$ (resp. $\lambda$ ) the $p$-adic valuation (resp. the degree) of $f(1-\gamma)$. They are respectivelly called $\mu$ invariant and $\lambda$-invariant.

Finally we denote by $\left(X_{\infty}^{\prime}\right)^{0}$ the maximal finite submodule of $X_{\infty}^{\prime}$.

P. Schneider prove (see $[S, \S 6$ lemma 1]) that the localisation kernels can be described as co-descent modules.

Theorem 3.1. For $i \in \mathbb{Z}$ and $i \neq 0$, we have a canonical isomorphism:

$$
\amalg_{S}^{2}\left(F, \mathbb{Z}_{p}(i+1)\right) \simeq\left(X_{\infty}^{\prime}(i)\right)_{\Gamma \times \Delta} .
$$


Remark. For $i=0$, the co-invariant $\left(X_{\infty}^{\prime}\right)_{\Gamma}$ is not isomorphic to the $p$-part of the $p$-class group $C l\left(\mathcal{O}_{E}^{\prime}\right)\{p\}$; it has been described by J.-F. Jaulent in logarithmic terms and it is isomorphic to the logarithmic class group $\widetilde{\mathcal{C}} \ell(E)$ (cp. [J2]). The Gross conjecture asserts that $\widetilde{\mathcal{C}} \ell(E)$ is finite.

Using Schneider's theorem, we can describe the extension maps in $F_{\infty} / F$ (see [N3] or [LMN]). For all $m \geq n \geq 0$, we denote $\gamma^{p^{n}}-1$ by $\omega_{n}$ and $\omega_{m} / \omega_{n}$ by $\nu_{m, n}$. Consider the natural morphisms $i_{m, n}$ :

$$
\begin{aligned}
X_{\infty}^{\prime}(i)_{\Gamma_{n}} & \rightarrow X_{\infty}^{\prime}(i)_{\Gamma_{m}} \\
x \bmod \omega_{n} & \mapsto \nu_{m, n} x \bmod \omega_{m} .
\end{aligned}
$$

We have a commutative diagram (with natural map for étale wild kernels):

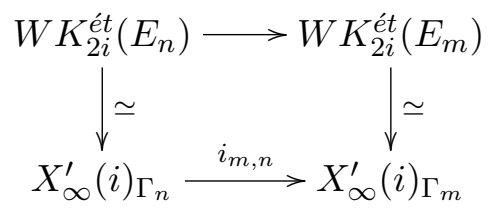

Proposition 3.1. For $i \geq 1$, and for $n$ sufficiently large, we have a canonical isomorphism:

$$
\operatorname{Cap}_{i}\left(E_{n}\right) \simeq\left(X_{\infty}^{\prime}\right)^{0}(i)
$$

As an easy consequence we find:

Corollary 3.1. For $i \geq 1$, and for all $m \geq n \gg 0$ :

$$
\operatorname{im}\left(\operatorname{Cap}_{i}\left(E_{n}\right) \rightarrow \operatorname{Cap}_{i}\left(E_{m}\right)\right) \simeq p^{m-n}\left(X_{\infty}^{\prime}\right)^{0}(i) .
$$

Proof. It follows from the description of the extension map and the fact that $\Gamma_{n}$ acts trivially on $\left(X_{\infty}^{\prime}\right)^{0}(i)$ for $n \gg 0$.

We can also describe the cokernel of the extension map. However we have to suppose that the $\mu$-invariant of $X_{\infty}^{\prime}$ is trivial. This is true when the extension $E / \mathbb{Q}$ is abelian (cf. [FW]); it is conjectured to be true for all number fields. In that case $X_{\infty}^{\prime}$ is finitely generated over $\mathbb{Z}_{p}$.

Proposition 3.2. Assume that $\mu$ is trivial. For all $m \geq n \gg 0$, we have the equality:

$$
\operatorname{im}\left(W K_{2 i}^{e ́ t}\left(E_{n}\right) \rightarrow W K_{2 i}^{e ́ t}\left(E_{m}\right)\right)=p^{m-n}\left(W K_{2 i}^{e ́ t}\left(E_{m}\right)\right)
$$

Proof. Let $T=1-\gamma$ thus $\Lambda \simeq \mathbb{Z}_{p}[[T]]$. Since $\mu=0$, we can assume that the characteristic polynomial $f(T)$ is a distinguished polynomial. There exists an integer $r \geq 0$, such that the distinguished polynomial $g(T)=\omega_{r}(T) f(T)$ annihilates $X_{\infty}^{\prime}$. Now we use a classical computation in Iwasawa theory. For $n$ sufficiently large we have

$$
(1+T)^{p^{n-1}} \equiv 1 \bmod (g(T), p) .
$$


Raising to the $p$-th power gives

$$
(1+T)^{p^{n}} \equiv 1 \bmod \left(g(T), p^{2}\right)
$$

Hence

$$
\begin{aligned}
\nu_{n+1, n} & =\sum_{i=0}^{p-1}(1+T)^{i p^{n}} \\
& \equiv p+p^{2} h(T) \bmod g(T) \\
& \equiv p(1+p h(T)) \bmod g(T)
\end{aligned}
$$

where $h(T) \in \Lambda$.

By induction we easily see that for $m \geq n$ there exists an invertible element $u_{m}(T) \in \Lambda$ such that $\nu_{m, n} \equiv p^{m-n} u_{m, n}(T) \bmod g(T)$. Hence we have $\operatorname{im}\left(i_{m, n}\right)=p^{m-n}\left(X_{\infty}^{\prime}\right)(i)_{\Gamma_{n}}$. Finally the proposition follows from Schneider's isomorphism.

We can now conclude:

Proposition 3.3. Assume that the $\mu$-invariant of $X_{\infty}^{\prime}$ is trivial. For all $i \geq 1$ and for all $n$ sufficiently large the exact sequence (3.1):

$$
0 \rightarrow \operatorname{Cap}_{i}\left(F_{n}\right) \rightarrow W K_{2 i}^{e ́ t}\left(F_{n}\right) \rightarrow W K_{2 i}^{e ́ t}\left(F_{\infty}\right)^{\Gamma_{n}} \rightarrow 0,
$$

is a split exact sequence of abelian groups.

Proof. First we reduce to the case of a number field containing the roots of unity of order $p$. Indeed the action of the semi-simple algebra $\mathbb{Z}_{p}[\Delta]$ on a finite abelian $p$-group keeps the direct summands. Then it is sufficient to prove that

$$
0 \rightarrow \operatorname{Cap}_{i}\left(E_{n}\right) \rightarrow W K_{2 i}\left(E_{n}\right) \rightarrow W K_{2 i}^{e ́ t}\left(E_{\infty}\right)^{\Gamma_{n}} \rightarrow 0
$$

is a split exact sequence of abelian groups to get the result.

Now choose an integer $r$ sufficiently large. Then for all $h \geq 0$ there is a commutative diagram (with natural maps):

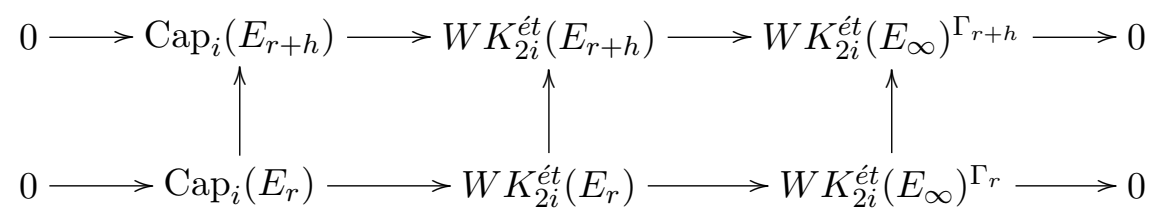

The vertical right arrow is injective. Hence by the snake lemma:

$$
\operatorname{coker}\left(\operatorname{Cap}_{i}\left(E_{r}\right) \rightarrow \operatorname{Cap}_{i}\left(E_{r+h}\right)\right) \hookrightarrow \operatorname{coker}\left(W K_{2 i}^{e ́ t}\left(E_{r}\right) \rightarrow W K_{2 i}^{e ́ t}\left(E_{r+h}\right)\right) \text {. }
$$

Corollary 3.1 gives a description for the left cokernel and proposition 3.2 (we assume that $\mu=0$ ) gives a description for the right cokernel. Let $e$ be 
an integer such that $p^{e}$ annihilates $\left(X_{\infty}^{\prime}\right)^{0}$ and $n=r+e$. For all $h$, with $0 \leq h \leq e$ we have:

$$
\operatorname{Cap}_{i}\left(E_{n}\right) / p^{h} \hookrightarrow W K_{2 i}^{e ́ t}\left(E_{n}\right) / p^{h} .
$$

Thus by lemma 1.1 the abelian $\operatorname{group} \operatorname{Cap}_{i}\left(E_{n}\right)$ is a direct summand in $W K_{2 i}^{e ́ t}\left(E_{n}\right)$.

It is well-known (cp.[KM] or [N3]) that co-descent holds for the wild kernels in the cyclotomic $\mathbb{Z}_{p}$-extension. In other words the norm map induces a canonical isomorphism for all $m \geq n \geq 0$ :

$$
W K_{2 i}^{e ́ t}\left(F_{m}\right)_{G_{m, n}} \simeq W K_{2 i}^{e ́ t}\left(F_{n}\right) .
$$

Although the extension map does not induce an isomorphism we have a non canonical Galois descent in the cyclotomic $\mathbb{Z}_{p}$-extension:

Proposition 3.4. Assume that $\mu=0$. Then for all $m \geq n \gg 0$, the groups $W K_{2 i}^{\text {ét }}\left(F_{m}\right)^{G_{m, n}}$ and $W K_{2 i}^{e ́ t}\left(F_{n}\right)$ are isomorphic as abelian groups.

Proof. Choose $n$ large and $m \geq n$. We have $\operatorname{Cap}_{i}\left(F_{m}\right)^{G_{m, n}}=\operatorname{Cap}_{i}\left(F_{m}\right)$. Consider the short exact sequence of $\mathbb{Z}_{p}\left[G_{m, n}\right]$-modules:

$$
0 \rightarrow \operatorname{Cap}_{i}\left(F_{m}\right) \rightarrow W K_{2 i}^{e ́ t}\left(F_{m}\right) \rightarrow W K_{2 i}^{e ́ t}\left(F_{\infty}\right)^{\Gamma_{m}} \rightarrow 0
$$

The snake lemma yields the long exact sequence:

$$
\begin{aligned}
0 \rightarrow \operatorname{Cap}_{i}\left(F_{m}\right) \rightarrow W K_{2 i}^{e ́ t}\left(F_{m}\right)^{G_{m, n}} & \rightarrow W K_{2 i}^{e ́ t}\left(F_{\infty}\right)^{\Gamma_{n}} \\
& \rightarrow \operatorname{Cap}_{i}\left(F_{m}\right)_{G_{m, n}} \rightarrow W K_{2 i}^{e ́ t}\left(F_{m}\right)_{G_{m, n}} \rightarrow \ldots
\end{aligned}
$$

Furthermore we have the commutative diagram:

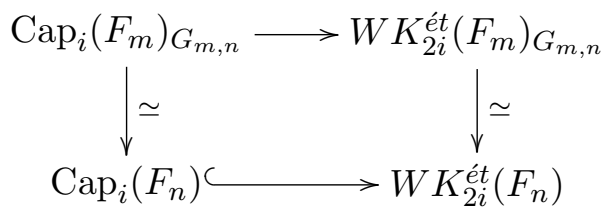

where the vertical maps are induced by the norm.

Hence the map $\operatorname{Cap}_{i}\left(F_{m}\right)_{G_{m, n}} \rightarrow W K_{2 i}^{e ́ t}\left(F_{m}\right)_{G_{m, n}}$ is injective. Thus we have a short exact sequence:

$$
0 \rightarrow \operatorname{Cap}_{i}\left(F_{m}\right) \rightarrow W K_{2 i}^{e ́ t}\left(F_{m}\right)^{G_{m, n}} \rightarrow W K_{2 i}^{e ́ t}\left(F_{\infty}\right)^{\Gamma_{n}} \rightarrow 0 .
$$

On the other hand $\operatorname{Cap}_{i}\left(F_{m}\right)$ is a direct summand in $W K_{2 i}^{e ́ t}\left(F_{m}\right)$. Hence it is a direct summand in the subgroup $W K_{2 i}^{e ́ t}\left(F_{m}\right)^{G_{m, n}}$ so (3.2) is a split 
exact sequence of abelian groups. Thus we have the isomorphisms of abelian groups:

$$
\begin{aligned}
W K_{2 i}^{e ́ t}\left(F_{m}\right)^{G_{m, n}} & \simeq \operatorname{Cap}_{i}\left(F_{m}\right) \oplus W K_{2 i}^{e ́ t}\left(F_{\infty}\right)^{\Gamma_{n}} \\
& \simeq \operatorname{Cap}_{i}\left(F_{n}\right) \oplus W K_{2 i}^{e ́ t}\left(F_{\infty}\right)^{\Gamma_{n}} \\
& \simeq W K_{2 i}^{e ́ t}\left(F_{n}\right) .
\end{aligned}
$$

Finally let us recall a descprition for the groups $W K_{2 i}^{e ́ t}\left(E_{\infty}\right)(\mathrm{cp} .[\mathrm{N} 3])$.

Proposition 3.5. Assume that $\mu$ is trivial. For all $i \geq 1$ we have:

$$
W K_{2 i}^{e ́ t}\left(E_{\infty}\right) \simeq\left(\mathbb{Q}_{p} / \mathbb{Z}_{p}\right)^{\lambda}
$$

as abelian group.

Proof. Since the groups $W K_{2 i}^{e ́ t}\left(E_{n}\right)$ are finite $(i \geq 1)$ the sequence $\left\{\omega_{n}\right\}_{n \geq 1}$ is an admissible sequence for the $\Lambda$-torsion module $X_{\infty}^{\prime}(i)$. Hence we have

$$
\beta\left(X_{\infty}^{\prime}(i)\right) \simeq \lim _{\rightarrow}\left(X_{\infty}^{\prime}(i)\right)_{\Gamma_{n}}=W K_{2 i}^{e ́ t}\left(E_{\infty}\right)
$$

where $\beta\left(X_{\infty}^{\prime}(i)\right)$ denotes the co-adjoint of $X_{\infty}^{\prime}(i)$.

Since we suppose $\mu=0$ the sequence $\left\{p^{n}\right\}_{n \geq 1}$ is also an admissible sequence for $X_{\infty}^{\prime}(i)$, whence

$$
\beta\left(X_{\infty}^{\prime}(i)\right) \simeq \lim _{\rightarrow}\left(X_{\infty}^{\prime}(i)\right) / p^{n}=\left(\mathbb{Q}_{p} / \mathbb{Z}_{p}\right)^{\lambda} .
$$

Remark. The results of this section are true for any finitely generated torsion $\Lambda$-module $X$ : assume that $X$ has a trivial $\mu$-invariant and that $X_{\Gamma_{n}}$ is finite for all $n \gg 0$, then the sequence

$$
0 \rightarrow X^{0} \rightarrow X_{\Gamma_{n}} \rightarrow\left(\lim _{\longrightarrow} X_{\Gamma_{n}}\right)^{\Gamma_{n}} \rightarrow 0,
$$

is a split exact sequence of abelian groups for all $n \gg 0(\mathrm{cp}$. [V, Théorème 3.1.8]).

\section{Capitulation for odd $K$-groups}

In the previous section we have shown that for $n$ sufficiently large the capitulation kernel is a direct summand in a subgroup of $K_{2 i}^{e ́ t}\left(\mathcal{O}_{F_{n}}^{S}\right)$. In this final section we prove that the capitulation kernel is still a direct summand in the entire group $K_{2 i}^{e ́ t}\left(\mathcal{O}_{F_{n}}^{S}\right)$.

Since Tate's works on $K_{2}$, many relations between the wild kernels and the class group have been highlighted. The following proposition shows that the deviation between the $p$-quotients of the wild kernels and the $p$-class group is asymptotically trivial in the cyclotomic $\mathbb{Z}_{p}$-extension. 
Assume that $E$ contains the roots of unity of order $p^{n}$. At the end of section 2 we contructed the canonical map:

$$
f_{(i, E, S)}:=W K_{2 i}^{e ́ t}(E) / p^{n} \rightarrow C l\left(\mathcal{O}_{E}^{S}\right) / p^{n}(i), \text { for all } i \geq 1 .
$$

This morphism could be surjective and not bijective for any set of primes containing $S_{p} \cup S_{\infty}$ : for example let $E$ be the Hilbert class field of $\mathbb{Q}\left(\mu_{37}\right)$. For the irregular prime number $p=37$ and for all $i \geq 1$, the wild kernels $W K_{2 i}^{e ́ t}(E)$ are cyclic while the class group $C l\left(\mathcal{O}_{E}^{\prime}\right)$ is trivial.

However the map $f_{(i, E, S)}$ is asymptotically bijective.

Proposition 4.1. Let $E$ be a number field containing $\mu_{p}$ and assume that the $\mu$ invariant of $X_{\infty}^{\prime}$ is trivial. Let $S$ be a set of primes containing the primes above $p$ and the infinite primes.

For all $h \geq 1$ there exists an integer $N$ such that for all $n \geq N$ and for all $i \geq 1$ the map $f_{\left(i, E_{n}, S\right)}$ yields the isomorphism:

$$
W K_{2 i}^{e ́ t}\left(E_{n}\right) / p^{h} \simeq \operatorname{Cl}\left(\mathcal{O}_{E_{n}}^{S}\right) / p^{h}(i) .
$$

Proof. We fix a positive integer $h$ and a set of primes $S$ as above. By Proposition 2.4 for all $n$ larger than a fixed integer $N$ the map

$$
W K_{2 i}^{e ́ t}\left(E_{n}\right) / p^{h} \rightarrow C l\left(\mathcal{O}_{E_{n}}^{S}\right) / p^{h}(i) \text { is onto. }
$$

Let us compute the order of both groups for $n \gg 0$. Since $W K_{2 i}^{e ́ t}\left(E_{\infty}\right)^{\Gamma_{n}}$ is finite we have

$$
W K_{2 i}^{e ́ t}\left(E_{\infty}\right)^{\Gamma_{n}} / p^{h} \simeq p^{h} W K_{2 i}^{e ́ t}\left(E_{\infty}\right)^{\Gamma_{n}}, \text { as abelian groups. }
$$

By Proposition 3.5 we have for $n \gg 0$ :

$$
p^{h} W K_{2 i}^{e ́ t}\left(E_{\infty}\right)^{\Gamma_{n}} \simeq \bigoplus_{k=1}^{\lambda} \mathbb{Z} / p^{h}, \text { as abelian groups. }
$$

Hence by Proposition 3.3 we see that

$$
W K_{2 i}^{e ́ t}\left(E_{n}\right) / p^{h} \simeq\left(X_{\infty}^{\prime}\right)^{0} / p^{h} \oplus \bigoplus_{k=1}^{\lambda} \mathbb{Z} / p^{h} \text {, as abelian groups. }
$$

On the other hand, since $S$ contains the primes above $p$, it is well known that $X_{\infty}^{S}:=\lim _{\longleftarrow} C l\left(\mathcal{O}_{E_{m}}^{S}\right)\{p\}=X_{\infty}^{\prime}$, independent of $S$.

Theorem $\overleftarrow{0.1}$ is still true for $S$-class groups (cp. [GJ]), so for $n \gg 0$ we have

$$
C l\left(\mathcal{O}_{E_{n}}^{S}\right) / p^{h} \simeq\left(X_{\infty}^{\prime}\right)^{0} / p^{h} \oplus \bigoplus_{k=1}^{\lambda} \mathbb{Z} / p^{h}, \text { as abelian groups. }
$$

Hence $W K_{2 i}^{e ́ t}\left(E_{n}\right) / p^{h}$ and $C l\left(\mathcal{O}_{E_{n}}^{S}\right) / p^{h}$ have the same order for $n$ sufficiently large and the canonical surjection

$$
W K_{2 i}^{e ́ t}\left(E_{n}\right) / p^{h} \rightarrow C l\left(\mathcal{O}_{E_{n}}^{S}\right) / p^{h}(i)
$$


is a bijection.

We may deduce an asymptotic rank formula for the wild kernels:

Corollary 4.1. Under the assumptions of Proposition 4.1 we have:

$$
\operatorname{dim}_{\mathbb{F}_{p}}\left(W K_{2 i}^{e ́ t}\left(E_{n}\right) / p\right)=\operatorname{dim}_{\mathbb{F}_{p}}\left(C l\left(\mathcal{O}_{E_{n}}^{S}\right) / p\right) \text {, for } n \text { large. }
$$

In [N1, Corollaire 5.7] (see also [KC, Corollary 3.3]), the author (assuming Leopoldt's conjecture) gives the rank formula:

$$
\operatorname{dim}_{\mathbb{F}_{p}}\left(W K_{2}^{\text {ét }}(E) / p\right)=\operatorname{dim}_{\mathbb{F}_{p}}\left(C l\left(\mathcal{O}_{E}^{\prime}\right) / p\right)+\operatorname{dim}_{\mathbb{F}_{p}}\left(W_{E} \cap p \mathcal{T}_{E} / p W_{E}\right),
$$

where

- $\mathcal{T}_{E}$ denotes the $\mathbb{Z}_{p}$-torsion of $\left(G_{E}^{S_{p}}\right)^{a b}$, and

- $W_{E} \simeq \Pi_{\mathfrak{p} \mid p} \mu\left(E_{\mathfrak{p}}\right) / \mu(E)$.

By Corollary 4.1 the $p$-rank of the wild kernel and the $p$-rank of the $p$-class group are the same for $n \gg 0$. Thus the group $W_{E_{n}} \cap p \mathcal{T}_{E_{n}} / p W_{E_{n}}$ is trivial (i.e. there is an injection $\left.W_{E_{n}} / p \hookrightarrow \mathcal{T}_{E_{n}} / p\right)$.

We can now prove the analogue of Theorem 0.1 for even étale $K$-groups.

Theorem 4.1. Let $S$ be a finite set of primes containing the primes above $p$ and the infinite primes. Assume that the $\mu$-invariant of the $\Lambda$-module $X_{\infty}^{\prime}$ is trivial.

Then for all $i \geq 1$ and for all $n$ sufficiently large the exact sequence (1.1):

$$
0 \rightarrow \operatorname{Cap}_{i}\left(F_{n}\right) \rightarrow K_{2 i}^{e ́ t}\left(\mathcal{O}_{F_{n}}^{S}\right) \rightarrow K_{2 i}^{e ́ t}\left(\mathcal{O}_{F_{\infty}}^{S}\right)^{\Gamma_{n}} \rightarrow 0
$$

is a split exact sequence of abelian groups.

Proof. As in the previous section it is sufficient to prove the result for the number field $E=F\left(\mu_{p}\right)$.

Let $p^{e}$ be the order of $\left(X_{\infty}^{\prime}\right)^{0}$. Let $h$ be a positive integer, with $0 \leq h \leq e$. Then for $n$ large:

(1) the field $E_{n}$ contains the roots of unity of order $p^{h}$.

(2) the group $\operatorname{Cap}_{i}\left(E_{n}\right)$ is a direct summand in $W K_{2 i}^{\text {ét }}\left(E_{n}\right)$.

(3) the canonical map $W K_{2 i}^{e ́ t}\left(E_{n}\right) / p^{h} \simeq C l\left(\mathcal{O}_{E_{n}}^{S}\right) / p^{h}(i)$ is an isomorphism.

Points (2) and (3) follow from the assumption $\mu=0$.

Using points (1) and (3) and Proposition 2.2 we can write the commutative diagram

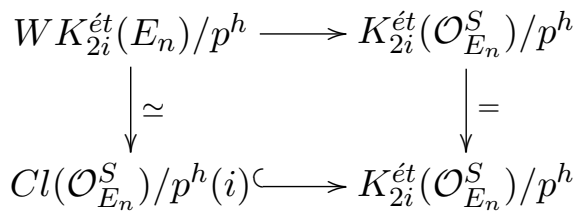


The left vertical map is bijective : it follows from Proposition 4.1. Thus the top horizontal arrow, induced by the inclusion, is injective.

On the other hand point (2) implies that $\operatorname{Cap}_{i}\left(E_{n}\right) / p^{h} \hookrightarrow W K_{2 i}^{e ́ t}\left(E_{n}\right) / p^{h}$. Hence we have:

$$
\operatorname{Cap}_{i}\left(E_{n}\right) / p^{h} \hookrightarrow K_{2 i}^{e ́ t}\left(\mathcal{O}_{E_{n}}^{S}\right) / p^{h}
$$

We finally use Lemma 1.1 to conclude that $\operatorname{Cap}_{i}\left(E_{n}\right)$ is a direct summand in the abelian group $K_{2 i}^{e ́ t}\left(\mathcal{O}_{E_{n}}^{S}\right)$.

The Galois co-descent holds for the étale $K$-groups in a $p$-ramified extension. Hence, as for the wild kernels, we have

Corollary 4.2. Under the assumptions of the previous theorem, for $n$ sufficiently large, and for all $m \geq n$, the groups $K_{2 i}^{\text {ét }}\left(\mathcal{O}_{E_{m}}^{S}\right)^{G_{m, n}}$ and $K_{2 i}^{\text {ét }}\left(\mathcal{O}_{E_{n}}^{S}\right)$ are isomorphic as abelian groups.

Finally, to get the result for the algebraic $K$-groups, we may use the Quillen-Lichtenbaum conjecture to identify algebraic and étale $K$-theory. This conjecture predicts that the Chern character yields the canonical isomorphism (see [Ko] and [W, Theorem 70]):

$$
K_{2 i}\left(\mathcal{O}_{F}^{T}\right) \otimes \mathbb{Z}_{p} \simeq K_{2 i}^{e ́ t}\left(\mathcal{O}_{F}^{T}[1 / p]\right)
$$

for all $i \geq 1$ and all finite sets of primes $T$.

Unpublished Voevodsky 's results on the Bloch-Kato conjecture for number fields seem prove this conjecture.

Theorem 4.2. Let $p$ be an prime number and $T$ be a finite set of primes of a number field $F$ containing $\sqrt{-1}$ if $p=2$. Let $F_{\infty}$ be the cyclotomic $\mathbb{Z}_{p}$-extension of $F$ with finite layers $F_{n}$ and assume that the $\mu$-invariant of the $\Lambda$-module $\lim _{\longleftarrow} C l\left(\mathcal{O}_{F\left(\mu_{p^{n}}\right)}^{\prime}\right)\{p\}$ is trivial. Let $i$ be a non-negative integer. Then, assuming the Quillen-Lichtenbaum conjecture, for $n$ large the capitulation kernel $\operatorname{Cap}_{i}\left(F_{n}\right)$ in $F_{\infty}$ is a direct summand in the abelian group $K_{2 i}\left(\mathcal{O}_{F_{n}}^{T}\right)\{p\}$.

Remark. We can wonder if the result still holds for the even $K$-groups of the fields $F_{n}$ (instead of its ring of integers). For $i=0$ the answer is trivial. For $i \geq 1$ it is well known that the étale wild kernels are isomorphic to the divisible part of $K_{2 i}\left(F_{n}\right)\{p\}$ (we recall that $p$ is odd and $\sqrt{-1} \in F$, if $p=2)$. Thus the capitulation kernel is contained in the divisible part of $K_{2 i}\left(F_{n}\right)\{p\}$ and it can not be a direct summand (except if it is trivial).

\section{References}

[AM] J. Assim \& A. Movahnedi, Bounds for étale Capitulation Kernels. K-theory, 33 (2004), 199-213.

[Ba] G. BANASZAK, Generalization of the Moore exact sequence and the wild kernel for higher K-groups. Compositio Math., 86 (1993), 281-305. 
[DF] W. Dwyer \& E. Friedlander, Algebraic and étale K-theory. Trans. Amer. Soc. 247 (1985), 247-280.

[FW] B. FERrero \& L. WAShington, The Iwasawa invariant $\mu_{p}$ vanishes for abelian number fields. Ann. Math. 109 (1979), 377-395.

[GJ] M. Grandet \& J.-F. Jaulent, Sur la capitulation dans les $\mathbb{Z}_{\ell}$-extensions. J. reine angew. Math. 362, 213-217.

[Iw] K. IwaSAWA, On $\mathbb{Z}_{\ell}$-extensions of algebraic number fields. Ann. Math. 98 (1973), 243326.

[J1] J.-F. Jaulent, Sur le noyau sauvage des corps de nombres. Acta Arith. 67 (1994), no.4, $335-348$.

[J2] J.-F. Jaulent, Théorie $\ell$-adique globale du corps de classes. J. Théor. Nombres Bordeaux 10 (1998), 355-397.

[JM] J.-F. JaUlent \& A. Michel, Approche logarithmique des noyaux étales sauvages des corps de nombres. J. Number Theory 120 (2006), no. 1, 72-91.

[Ka] B. KaHn, Descente galoisienne et $K_{2}$ des corps de nombres. K-theory 7 (1993), 55-100.

[KC] K. Krammer \& A. Candiotti, On $K_{2}$ and $\mathbb{Z}_{l}$ extensions of number fields. Amer. J. Math. 100 (1978), 177-196.

[KM] M. Kolster \& A. MovahHedi, Galois co-descent for étale wild kernels and capitulation. Ann. Inst. Fourier 50 (2000), 35-65.

[Ko] M. Kolster, $K$-theory and arithmetic. Contemporary developments in algebraic $K$ theory, ICTP Lect. Notes, XV, Abdus Salam Int. Cent. Theoret. Phys. Trieste, (2004).

[Ku] L. V. Kuz'min, The Tate module for algebraic number fields. Math. USSR Izv.,6, No. 2 (1972), 263-361.

[LMN] M. Le Floc'h, A. Movahhedi \& T. Nguyen Quang Do, On capitulation cokernels in Iwasawa theory. Amer. Journal of Mathematics, 127 (2005), 851-877.

[N1] T. NGuyen Quang Do, Sur la $\mathbb{Z}_{p}$-torsion de certains modules galoisiens. Ann. Inst. Fourier 36, no. 2 (1986), 27-46.

[N2] T. Nguyen Quang Do, Analogues supérieurs du noyau sauvage. Journal de Théorie des Nombres de Bordeaux 4 (1992), 263-271.

[N3] T. NGuyen Quang Do, Théorie d'Iwasawa des noyaux sauvages étales d'un corps de nombres. Publications Math. de la Faculté des Sciences de Besançon (2002).

[NSW] J. Neukirch, A. Schmidt \& K. Wingberg, Cohomology of number fields. Springer Verlag, Berlin (2000).

[S] P. SCHNEIDER, Über gewisse Galoiscohomologiegruppen. Math. Z. 168, 181-205 (1979).

[Ta] J. TATE, Relations between $K_{2}$ and Galois cohomology. Invent. Math. 36 (1976), 257-274.

[V] R. VALIDIRE, Capitulation des noyaux sauvages étales. Thèse de l'Université de Limoges, (2008).

[W] C. Weibel, Algebraic K-theory of rings of integers in local and global fields. Handbook of $K$-theory Vol. 1, 2, 139-190, Springer, Berlin, (2005).

\author{
Romain VALIDIRE \\ XLIM DMI - UMR CNRS 6172 \\ 123, avenue Albert Thomas \\ 87060 LIMOGES CEDEX (France). \\ E-mail: romain.validire@gmail.com \\ URL: http://www.math.u-bordeaux.fr/A2X/
}

